\title{
HIERARCHICAL ANALYSIS OF MOLECULAR SPECTRA
}

\author{
Michael J. Davis \\ Theoretical Chemistry Group, Chemistry Division, Argonne National Laboratory
}

A novel representation of molecular spectra in terms of hierarchical trees has proven to be an important aid for the study of many significant problems in gas-phase chemical dynamics. Trees are generated from molecular spectra by monitoring the changes that occur in a spectrum as resolution is changed in a continuous manner. A tree defines a genealogy among all lines of a spectrum. This allows for a detailed understanding of the assignment of features of a spectrum that may be difficult to obtain any other way as well as an understanding of intramolecular energy transfer time scales, mechanisms, and pathways. The methodology has been applied to several problems: transition state spectroscopy, intramolecular energy transfer in highly excited molecules, high-resolution overtone spectroscopy, and the nature of the classical-quantum correspondence when there is classical chaos ("quantum chaos"). This method has been developed by Michael J. Davis at Argonne National Laboratory with the support of the Division of Chemical Sciences.

\section{DISCLAIMER}

\begin{abstract}
This report was prepared as an account of work sponsored by an agency of the United States Government. Neither the United States Government nor any agency thereof, nor any of their employees, makes any warranty, express or implied, or assumes any legal liability or responsibility for the accuracy, completeness, or usefulness of any information, apparatus, product, or process disclosed, or represents that its use would not infringe privately owned rights. Reference herein to any specific commercial product, process, or service by trade name, trademark, manufacturer, or otherwise does not necessarily constitute or imply its endorsement, recommendation, or favoring by the United States Government or any agency thereof. The views and opinions of authors expressed herein do not necessarily state or reflect those of the United States Government or any agency thereof.
\end{abstract}

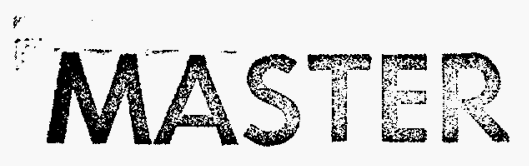




\title{
HIERARCHICAL ANALYSIS OF MOLECULAR SPECTRA
}

\author{
Michael J. Davis \\ Theoretical Chemistry Group, Chemistry Division, Argonne National Laboratory
}

Many combustion processes involve multistep reaction mechanisms that include reactions involving highly excited intermediates that can be modeled as unimolecular reactions. In the last two decades two developments have aided in the study of unimolecular reactions. The first is the development of spectroscopic techniques that can probe highly excited molecules. The second involves the increased power of computers, which allows the theoretical calculation of both better potential energy surfaces and highly excited vibrational eigenstates. These developments allow for a better understanding of the dynamics of highly excited molecules, and thus the energy transfer processes that lead to the dissociation of highly excited molecules, but the data from both the experimental and theoretical developments may be very complicated.

Over the last several years we have developed an approach to the study of both complicated molecular spectra and vibrational eigenstates that allows for a better understanding of the energy transfer implied in both the spectroscopy and eigenstates. Hierarchical trees can be generated from a spectrum by following the changes that occur as resolution is changed in a continuous manner. These trees can then be analyzed using techniques developed in the classification and multivariate analysis literature. They can also be used to map out in detail the energy transfer pathways and time scales when a theoretical version of a spectrum generated from the eigenstates is available.

The attached figure shows the results of our analysis as applied to an experimental excitation spectrum of $\mathrm{NO}_{2}$, which is quite complicated due to vibronic interactions. The top part of the plot shows the experimental spectrum and the bottom part shows our analysis. By viewing a spectrum at increasing resolution (left side, DE) one can observe changes in the spectrum that can be analyzed with a hierarchical tree shown at the bottom center. The tree is color coded based on time scales and can be matched with the lower resolution spectra on the left. This increase in resolution is connected 
to increased times by the uncertainty principle (see the bottom). By studying the tree at increasing times (right side), one can understand the nature of the intramolecular dynamics as it unfolds.

Over the last two years we have undertaken applications in several areas of physical chemistry. One application has been to transition state spectroscopy, specifically the photodetachment spectrum of $\mathrm{OHCl}^{-}$. We were able to show how the metastable $\mathrm{OHCl}$ neutral moved in the vicinity of the transition state. Another application has been to the study of IVR (intramolecular vibrational redistribution). Specifically, we have studied the experimental $3 v_{1}$ band of propyre, and have been able to assess the role of vibrational versus rotational couplings in the onset of IVR. Our results suggest that IVR for this excitation of propyne is dominated by vibrational couplings through at least 1 ns. Finally, we have used the hierarchical analysis to continue our study of the nature of quantum intramolecular energy transfer in the presence of classical chaos. We have been able to map out detailed energy transfer pathways for several small systems. Our studies also demonstrate how short time localized motion can be manifested in the long time intramolecular dynamics and further be manifested in molecular spectra at various levels of resolution. 


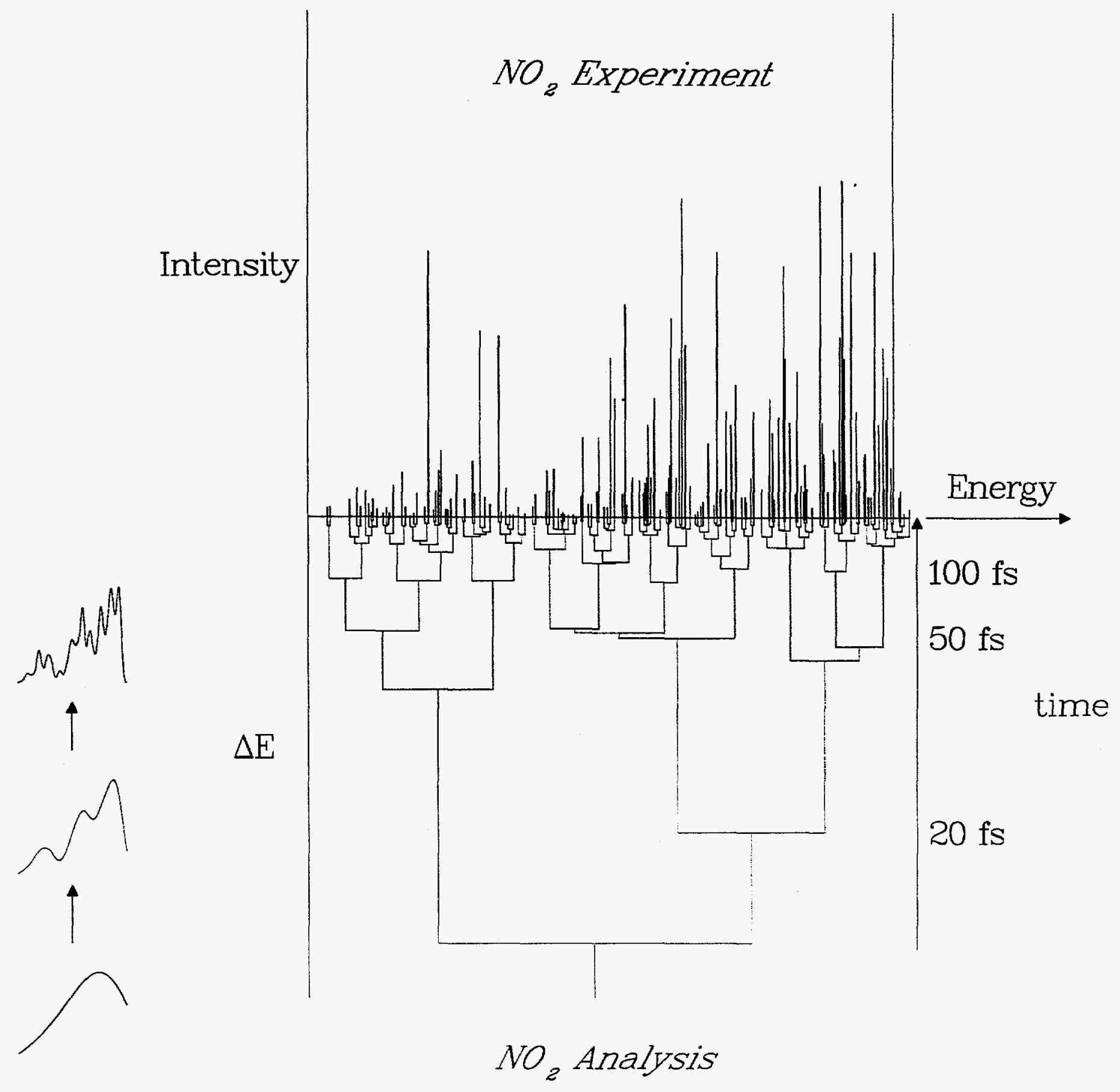

$\Delta \mathrm{E} \longrightarrow$ uncertainty principle $\longrightarrow$ time 


\section{DISCLAMMER}

Portions of this document may be illegible in electronic image products. Images are produced from the best available original document. 